\title{
The microenvironment in sporadic and neurofibromatosis type II-related vestibular schwannoma: the same tumor or different? A comparative imaging and neuropathology study
}

\author{
*Daniel Lewis, MRCS, MA,, Carmine A. Donofrio, MD, ${ }^{1,}$ Claire O'Leary, PhD, ${ }^{1,3} \mathrm{Ka}-$-loh Li, PhD, ${ }^{2}$ \\ Xiaoping Zhu, MD, PhD, ${ }^{2}$ Ricky Williams, ${ }^{1}$ Ibrahim Djoukhadar, PhD, FRCR, ${ }^{1}$ \\ Erjon Agushi, MBBS, ${ }^{2}$ Cathal J. Hannan, MRCS, ${ }^{1}$ Emma Stapleton, FRCS(ORL-HNS), ${ }^{4}$ \\ Simon K. Lloyd, MPhil, FRCS(ORL-HNS), ${ }^{4}$ Simon R. Freeman, FRCS(ORL-HNS), ${ }^{4}$ \\ Andrea Wadeson, BSc, ${ }^{1}$ Scott A. Rutherford, FRCS(SN), ${ }^{1}$ \\ Charlotte Hammerbeck-Ward, PhD, FRCS(SN), ${ }^{1}$ D. Gareth Evans, MD, FRCP, ${ }^{5}$ \\ Alan Jackson, PhD, FRCR, ${ }^{2}$ Omar N. Pathmanaban, PhD, FRCS(SN), ${ }^{1,6}$ Federico Roncaroli, MD, ${ }^{1,3}$ \\ Andrew T. King, FRCS(SN), ${ }^{1,7}$ and David J. Coope, PhD, FRCS(SN) ${ }^{1,3}$
}

'Department of Neurosurgery, Manchester Centre for Clinical Neurosciences, Salford Royal NHS Foundation Trust, Manchester Academic Health Science Centre; '2Division of Informatics, Imaging and Data Sciences, Wolfson Molecular Imaging Centre (WMIC), University of Manchester; ${ }^{3}$ Division of Neuroscience and Experimental Psychology, School of Biological Sciences, Faculty of Biology, Medicine and Health, University of Manchester; ${ }^{4}$ Department of Otolaryngology, Salford Royal NHS Foundation Trust, Manchester Academic Health Science Centre; ${ }^{5}$ Division of Evolution and Genomic Sciences, School of Biological Sciences, Faculty of Biology, Medicine and Health, University of Manchester; ${ }^{6}$ Division of Cell Matrix Biology \& Regenerative Medicine, School of Biological Sciences, Faculty of Biology, Medicine and Health, University of Manchester; and ${ }^{7}$ Division of Cardiovascular Sciences, School of Medical Sciences, Faculty of Biology, Medicine and Health, University of Manchester, United Kingdom

OBJECTIVE Inflammation and angiogenesis may play a role in the growth of sporadic and neurofibromatosis type 2 (NF2)-related vestibular schwannoma (VS). The similarities in microvascular and inflammatory microenvironment have not been investigated. The authors sought to compare the tumor microenvironment (TME) in sporadic and NF2-related VSs using a combined imaging and tissue analysis approach.

METHODS Diffusion MRI and high-temporal-resolution dynamic contrast-enhanced (DCE) MRI data sets were prospectively acquired in 20 NF2-related and 24 size-matched sporadic VSs. Diffusion metrics (mean diffusivity, fractional anisotropy) and DCE-MRI-derived microvascular biomarkers (transfer constant [Krans], fractional plasma volume, tissue extravascular-extracellular space $\left[\mathrm{V}_{\mathrm{e}}\right.$, longitudinal relaxation rate, tumoral blood flow) were compared across both VS groups, and regression analysis was used to evaluate the effect of tumor size, pretreatment tumor growth rate, and tumor NF2 status (sporadic vs NF2-related) on each imaging parameter. Tissues from 17 imaged sporadic VSs and a separate cohort of 12 NF2-related VSs were examined with immunohistochemistry markers for vessels (CD31), vessel permeability (fibrinogen), and macrophage density (Iba1). The expression of vascular endothelial growth factor (VEGF) and VEGF receptor 1 was evaluated using immunohistochemistry, Western blotting, and double immunofluorescence.

RESULTS Imaging data demonstrated that DCE-MRI-derived microvascular characteristics were similar in sporadic and NF2-related VSs. Krans $(p<0.001), v_{e}(p \leq 0.004)$, and tumoral free water content $(p \leq 0.003)$ increased with increasing tumor size and pretreatment tumor growth rate. Regression analysis demonstrated that with the exception of mean

\footnotetext{
ABBREVIATIONS $\mathrm{CBF}_{\mathrm{ET}}=$ absolute cerebral blood flow; $\mathrm{DCE}=$ dynamic contrast-enhanced; $\mathrm{DWI}=$ diffusion-weighted imaging; $F A=$ fractional anisotropy; $\mathrm{K}^{\text {trans }}=$ transfer constant; $\mathrm{MD}$ = mean diffusivity; NF2 = neurofibromatosis type $2 ; \mathrm{OD}=$ optical density; $\mathrm{R} 1 \mathrm{~N}=$ longitudinal relaxation rate; $\mathrm{SA}=$ surface area; $\mathrm{SRS}=$ stereotactic radiosurgery; TAM = tumor-associated macrophage; TME = tumor microenvironment; $\mathrm{v}_{\mathrm{e}}=$ tissue extravascular-extracellular space; VEGF = vascular endothelial growth factor; VEGFR-1 $=$ VEGF receptor $1 ; v_{p}=$ fractional plasma volume; $V S=$ vestibular schwannoma.

SUBMITTED December 8, 2019. ACCEPTED March 11, 2020.
}

INCLUDE WHEN CITING Published online May 29, 2020; DOI: 10.3171/2020.3.JNS193230.

${ }^{*}$ D.L. and C.A.D. share first authorship of this work. A.T.K. and D.J.C. share senior authorship of this work. 
diffusivity ( $p<0.001)$, NF2 status had no statistically significant effect on any of the imaging parameters or the observed relationship between the imaging parameters and tumor size $(p>0.05)$. Tissue analysis confirmed the imaging metrics among resected sporadic VSs and demonstrated that across all VSs studied, there was a close association between vascularity and $\mathrm{lba1}{ }^{+}$macrophage density $(r=0.55, p=0.002)$. VEGF was expressed by $\mathrm{lba1} 1^{+}$macrophages.

CONCLUSIONS The authors present the first in vivo comparative study of microvascular and inflammatory characteristics in sporadic and NF2-related VSs. The imaging and tissue analysis results indicate that inflammation is a key contributor to TME and should be viewed as a therapeutic target in both VS groups.

https://thejns.org/doi/abs/10.3171/2020.3.JNS193230

KEYWORDS vestibular schwannoma; neurofibromatosis type 2; NF2; inflammation; DCE-MRI; vascular endothelial growth factor; VEGF; oncology

$\mathrm{A}$ PPROXIMATELY $95 \%$ of vestibular schwannomas (VSs) occur as a unilateral, sporadic tumor. Bilateral VSs are, conversely, the hallmark of patients with the dominantly inherited tumor syndrome, neurofibromatosis type 2 (NF2). ${ }^{1}$ Sporadic and NF2-related VSs share biallelic inactivation of the NF2 gene, ${ }^{2-4}$ but they present with phenotypic differences. ${ }^{5,6}$ NF2-related VSs develop as multifocal tumors along cranial nerve VIII ${ }^{5,6}$ and are therefore difficult to remove. Sporadic VSs are challenging due to their often large size at presentation and their unpredictable growth rate.?

It is currently unknown if the tumor microenvironment (TME) differs in sporadic and NF2-related VSs. There is growing evidence that tumor-associated macrophages (TAMs) may play a role in the growth and progression of sporadic VS. ${ }^{8-10}$ Previous studies examined the inflammatory microenvironment in NF2-related tumors, ${ }^{11,12}$ but TME data in both VS groups are limited as lesions are often resected at different time points in their growth trajectory. $3,7,13-16$ Angiogenesis has also received increased attention as a potential therapeutic target in both sporadic and NF2-related tumors. ${ }^{17-20}$ Tumor microvasculature and tumor microstructure can be investigated and monitored with advanced MRI modalities such as dynamic contrastenhanced (DCE) MRI. ${ }^{21}$ DCE-MRI-derived microvascular parameters correlate well with tissue markers of permeability, microvessel surface area (SA), and cell density.., 22

In the present study we used both DCE-MRI and diffusion MRI to compare the microvascular and microstructural characteristics across a cohort of sporadic and NF2-related VSs and establish if their TMEs are similar. By studying the tissue of resected tumor specimens, we sought to establish to what extent DCE-MRI-derived imaging biomarkers reflect the inflammation and microvasculature in these tumors and understand the relationship between inflammation and angiogenesis in both VS groups.

\section{Methods}

\section{Study Design and Patient Population}

DCE-MRI data sets from two separate patient cohorts were used in this study. Between December 2015 and August 2018, 24 patients with sporadic VSs were recruited via the Manchester Skull Base Unit multidisciplinary team meeting at Salford Royal Hospital in Salford, United Kingdom. Fifteen of these patients were recruited under a combined $\left[{ }^{11} \mathrm{C}\right]-(\mathrm{R})$ PK-11195 PET and DCE-MRI study.
A further 9 patients were recruited under a separate ethics protocol and underwent only the MRI acquisition. All patients underwent at least two MRI acquisitions to establish tumor growth, including the study scan in 6 patients. The study MRI scan and the results of previous MRI were reviewed collectively by the multidisciplinary team and tumors were classified as static/shrinking, growing, or sufficiently large to preclude monitoring of growth and mandate early resection. This classification was based upon clinical decision, with patients with growing VSs recommended to undergo either microsurgery or stereotactic radiosurgery (SRS), and patients with static or shrinking tumors offered a period of observation. The median length of follow-up from diagnosis was 29 months (IQR 23-49 months) for the static/shrinking cohort, 12 months (IQR 8-25 months) for growing tumors, and 3 months (IQR 2-6 months) for the large tumor cohort, reflecting the early decision to treat these patients. Volumetric measurements of tumor size were made for both the study MRI scan and the preceding clinical scan to confirm different growth behaviors between the tumor cohorts. ${ }^{8}$

In addition, pretreatment DCE-MRI data from 12 patients with NF2 who had been recruited as part of a previous study at our institution were retrospectively analyzed. ${ }^{23}$ These patients had been recruited through the NF2 clinic in Manchester, United Kingdom, and following imaging, all patients underwent treatment with the anti-vascular endothelial growth factor (VEGF) antibody bevacizumab. All patients had proven NF2 with at least 1 VS demonstrating a high growth rate, defined as an increase of 4 $\mathrm{mm}$ or greater in the maximal transverse diameter over a 12-month period. ${ }^{23}$ Similar to the sporadic VS cohort, volumetric measurements of tumor size were made for both the study MRI scan and the preceding clinical scan. For all VSs studied, tumor extrameatal extension and the degree of tumor brainstem compression at the time of the study scan were reported using the Koos classification system. ${ }^{44}$

\section{Imaging Methods \\ MRI Acquisition}

For all patients, MRI data were acquired on a 1.5-T whole-body scanner using a dedicated head coil (Philips Achieva, Philips Medical Systems). A high-resolution 3D T1-weighted gradient echo sequence with full brain coverage (TE $3.2 \mathrm{msec}$, TR $8.6 \mathrm{msec}$, slice thickness 1.2 $\mathrm{mm}$ ) both before and after contrast was obtained for tumor delineation. Diffusion-weighted imaging (DWI) was 
obtained using 6 directions and $3 \mathrm{~B}$ values $(0,400$, and $800 \mathrm{sec} / \mathrm{mm}^{3}$ ), and DCE-MRI data were collected using a previously described dual-injection, dual-temporal-resolution technique, ${ }^{24}$ the details of which can be found in the Supplementary Methods.

\section{MRI Data Analysis}

Acquired DWI data for both the sporadic and NF2 patient cohorts were processed using the FSL 4.1 Diffusion Toolbox (http://www.fmrib.ox.ac.uk/fsl/) through a standard multistep procedure that incorporated both eddy current correction and brain extraction. ${ }^{25}$ Through this approach, voxel-wise maps of mean diffusivity (MD) and fractional anisotropy (FA) were generated. Voxel-wise maps of microvascular kinetic parameters and absolute cerebral blood flow $\left(\mathrm{CBF}_{\mathrm{ET}}\right)$ estimates were derived from the low-dose, high-temporal-resolution DCE-MRI data sets using the extended Tofts model ${ }^{21}$ and a previously developed T1-weighted early time points method, ${ }^{26}$ respectively. Individual tumors were manually delineated on the coregistered T1-weighted postcontrast image to create a tumor object mask. These object masks were then applied to the $\mathrm{CBF}_{\mathrm{ET}}$ map, kinetic parameter maps, and diffusion parameter maps (MD, FA) to allow derivation of whole tumor blood flow, mean kinetic parameter estimates (transfer constant $\left[\mathrm{K}^{\text {trans }}\right]$, tissue extravascular-extracellular space $\left[\mathrm{v}_{\mathrm{e}}\right]$, fractional plasma volume $\left[\mathrm{v}_{\mathrm{p}}\right]$, longitudinal relaxation rate $\left[R 1_{N}\right]$ ), and diffusion metrics $(\mathrm{MD}, \mathrm{FA})$. Individual tumor volumes were determined on postcontrast T1-weighted imaging. Further details are provided in the Supplementary Methods.

\section{Tissue Analysis}

Tissues from 17 sporadic tumors that had undergone DCE-MRI and a separate cohort of 12 previously resected NF2-related VSs were analyzed. Ethical approval was given for all tissue analyses. Frozen samples were collected for 10 patients (6 sporadic and 4 NF2-related VSs). Serial 5- $\mu \mathrm{m}$ sections were cut from each paraffin block and used for H \& E staining, immunoperoxidase immunohistochemistry, and immunofluorescence. Tissue sections were assessed quantitatively for TAM density (Iba1), microvessel area (CD31), vascular permeability (fibrinogen), and cellular proliferation (anti-Ki-67) using immunoperoxidase immunohistochemistry. Semiquantitative evaluation of VEGF and VEGF receptor 1 (VEGFR-1) was also performed. Western blot analysis on the 10 frozen specimens was used to confirm VEGF and VEGFR-1 protein expression. Double immunofluorescence staining for Iba1 and VEGF was performed on 8 paraffin sections (4 sporadic and 4 NF2-related VSs) to evaluate the VEGF expression in macrophages. Detailed protocols are described in Supplementary Methods.

For quantitative analysis, 20 representative images were taken at magnification $\times 20$ from the $\mathrm{H} \& \mathrm{E}$-stained sections and sections stained with Iba1, CD31, fibrinogen, VEGF, and VEGFR-1 for each case using a 3DHistech Pannoramic-250 microscope slide-scanner (3DHistech Ltd.). All images were analyzed using ImageJ software (https://imagej.nih.gov/ij/). Quantification was performed by two independent observers (D.L. and C.A.D.); in- terobserver agreement was greater than 95\% across all measurements, and results were further validated by an experienced neuropathologist (F.R.) by reviewing and quantifying random images from each case. Negative controls were performed by substituting the primary antibody with phosphate-buffered saline. Normal tonsil and appendix tissues served as positive controls for all primary antibodies tested (Supplementary Fig. S1). Detailed tissue analysis protocols are shown in the Supplementary Methods.

\section{Statistical Analysis}

The statistical program Stata (version 11, StataCorp LLC) was used for all statistical analyses. Normality and homogeneity of variance for all individual data parameters were assessed using the Shapiro-Wilk and Levene tests, respectively. For comparison of parametric diffusion and DCE-MRI-derived values between sporadic and NF2related VSs, a 2-tailed t-test was used. For nonparametric data (patient age, tumor size, annual adjusted growth rate) the Mann-Whitney U-test was used. The intertumor correlations between tumor size, imaging-derived parameters, and tissue-derived parameters are reported as Pearson's product-moment correlation coefficient (r), or Spearman's rho in the case of nonlinear associations. For linear associations, the results of linear regression are reported as adjusted $R^{2}$ estimates on included figures.

A 2-tailed t-test was used for comparison of parametric tissue-derived parameters (CD31 microvessel SA, fibrinogen optical density [OD], Ki-67 labeling index) between sporadic and NF2-related VSs and between high and low intratumoral $\mathrm{Iba}^{+}$areas. For comparison between sporadic VS growth groups (static, growing, large) a 1-way ANOVA with Bonferroni correction was used. Semiquantitative (VEGF, VEGFR-1) and categorical variables were compared using either Pearson's chi-square or Fisher's exact test. To evaluate the effect of tumor size, annual adjusted growth rate, and tumor NF2 status on imaging and tissue-derived parameters, simple univariate and multiple linear regression models, with tumor NF2 status as an interaction term, were used. Nonlinear associations were addressed through use of the cube root transform of tumor size and tumor annual adjusted growth rate in the regression models. Residual normality was analyzed using the Jarque-Bera test and by assessing the linearity of quantile-quantile plots. Heteroscedasticity was evaluated using White's test and by producing scatterplots of residuals versus predicted values. Multicollinearity between predictor variables was assessed through postregression testing and collinear variables were excluded.

\section{Results \\ Patient Population}

Details of the demographics of the 24 patients with sporadic VS and 12 NF2 patients included in the imaging study are presented in Table 1. The median age across the sporadic VS cohort was 55.3 years (IQR 42.2-62.1 years). Seventeen patients underwent resection, 2 were treated with SRS, and 5 had no treatment and are undergoing long-term follow-up. Eight tumors were classified as 
TABLE 1. Comparative table of patient demographics, DCE-MRI-derived microvascular parameters, and diffusion metrics between sporadic and NF2-related VSs

\begin{tabular}{|c|c|c|c|c|}
\hline Variable & Sporadic VS $(n=24)$ & NF2-Related VS $(n=20)$ & Mean Difference $(95 \% \mathrm{Cl})$ & p Value \\
\hline Median age (IQR), yrs & $55.3(42.2-62.1)$ & $24.0(20.5-29.0)$ & $-24.2(-32.6$ to -15.7$)$ & $<0.001$ \\
\hline Koos grade, $\mathrm{n}(\%)$ & & & & $0.89 \dagger$ \\
\hline II & $7(29)$ & $6(30)$ & & \\
\hline III & $10(42)$ & $7(35)$ & & \\
\hline IV & $7(29)$ & $7(35)$ & & \\
\hline Median VS size (range), $\mathrm{cm}^{3}$ & $1.82(0.34-18.67)$ & $2.51(0.20-17.87)$ & $0.20(-2.66$ to 3.06$)$ & 0.65 \\
\hline Median annual adjusted growth rate (IQR), $\mathrm{cm}^{3} / \mathrm{yr}$ & $0.62(0.09-1.29)$ & $0.75(0.23-2.24)$ & $0.16(-0.78$ to 1.11$)$ & 0.48 \\
\hline Mean tumor $\mathrm{K}^{\text {trans }}(\mathrm{SD})$, min $^{-1}$ & $0.12(0.05)$ & $0.11(0.04)$ & $-0.01(-0.04$ to 0.02$)$ & 0.49 \\
\hline Mean tumor $v_{p}(S D)$ & $0.05(0.02)$ & $0.05(0.02)$ & $-0.0006(-0.01$ to 0.01$)$ & 0.91 \\
\hline Mean tumor $\mathrm{v}_{\mathrm{e}}(\mathrm{SD})$ & $0.37(0.12)$ & $0.35(0.08)$ & $-0.02(-0.09$ to 0.04$)$ & 0.49 \\
\hline Mean TBF (SD), $\mathrm{ml} / 100 \mathrm{ml} / \mathrm{min}$ & $93.3(31.1)$ & $89.5(23.2)$ & $-3.78(-20.7$ to 13.2$)$ & 0.65 \\
\hline Mean tumor $\mathrm{R} 1_{\mathrm{N}}(\mathrm{SD}), \mathrm{sec}^{-1}$ & $0.68(0.12)$ & $0.76(0.17)$ & $0.07(-0.01$ to 0.16$)$ & 0.08 \\
\hline $\mathrm{MD}(\mathrm{SD}), \mathrm{mm}^{2} / \mathrm{sec} \times 10^{-3}$ & $0.67(0.09)$ & $0.80(0.12)$ & $0.14(0.07$ to 0.20$)$ & $<0.001$ \\
\hline $\mathrm{FA}(\mathrm{SD})$ & $0.39(0.06)$ & $0.36(0.05)$ & $-0.03(-0.07$ to -0.000003$)$ & 0.05 \\
\hline
\end{tabular}

Boldface type indicates statistical significance.

* The $p$ value was calculated using a 2-tailed t-test, or Mann-Whitney U-test in the case of nonparametric data.

$\dagger$ Differences in tumor Koos grade determined using Pearson's chi-square test.

static or shrinking $(\mathrm{n}=1), 12$ as growing, and 4 tumors as sufficiently large to preclude monitoring of growth and mandate early resection. Confirmatory volumetric measurements demonstrated that the growing tumors were significantly larger ( 2.28 vs $\left.0.62 \mathrm{~cm}^{3}, \mathrm{p}<0.001\right)$ and displayed a significantly higher annual adjusted growth rate compared with the static/shrinking group (0.77 vs $0.02 \mathrm{~cm}^{3} /$ year, $\mathrm{p}<0.001)$. The median size among the 4 large sporadic tumors was $13.0 \mathrm{~cm}^{3}$ (IQR $10.7-16.6 \mathrm{~cm}^{3}$ ), and while they were primarily treated due to large size at presentation, volumetric measurements confirmed growth in these tumors within the short follow-up period (median annual adjusted growth rate $=2.94 \mathrm{~cm}^{3} /$ year, IQR $1.70-5.25 \mathrm{~cm}^{3} /$ year). The NF2 patient cohort was significantly younger compared to patients with sporadic tumors $(\mathrm{p}<0.001)$; the median age was 24 years (IQR 20.5-29.0 years). Of the 12 NF2 patients, 4 had undergone previous resection of a contralateral VS. Median tumor size $\left(2.51 \mathrm{vs} 1.82 \mathrm{~cm}^{3}\right)$ and median annual adjusted growth rate $\left(0.75 \mathrm{vs} 0.62 \mathrm{~cm}^{3} /\right.$ year) were higher among NF2-related VSs compared to sporadic VSs, but these differences were not statistically significant (Table 1).

Features of the 17 sporadic VS and 12 NF2 patients whose tissue was used for analysis are detailed in Table 2. The NF2 patient cohort demonstrated significantly higher tumor volumes at the time of resection (median volume 8.43 vs $2.51 \mathrm{~cm}^{3}, 95 \%$ CI mean difference $=0.53-17.9$ $\mathrm{cm}^{3}$ ) and a nonsignificantly longer median period of radiological follow-up prior to resection (28 vs 13 months, 95\% CI mean difference $=-2$ to 37 months). Among resected sporadic tumors, 3 were classified as static, 10 as growing, and 4 tumors as sufficiently large to preclude monitoring of growth and mandate early resection. Across the resected NF2-related VS cohort there was a range of pretreatment tumor growth rates, and these rates were comparable to those of the resected sporadic VS cohort (median annual adjusted growth rate $=0.80$ vs $0.77 \mathrm{~cm}^{3} / y e a r, 95 \%$ CI mean difference $=-1.17$ to $1.65 \mathrm{~cm}^{3} /$ year).

\section{Imaging Data}

Comparative DCE-MRI and diffusion MR-derived imaging metrics between the sporadic and NF2-related VS cohorts are shown in Table 1. NF2-related VSs displayed significantly higher tumor MD values $(\mathrm{p}<0.001)$ and lower FA values $(\mathrm{p}=0.05)$ than sporadic tumors. There was no significant difference in any of the DCE-MRI-derived microvascular metrics between the two tumor groups $(\mathrm{p}$ $>0.05)$.

Mean tumor $\mathrm{K}^{\text {trans }}$ increased with increasing tumor size in both sporadic (rho $=0.83, \mathrm{p}<0.001$ ) and NF2-related $($ rho $=0.77, p<0.001)$ VSs (Fig. 1A). There were sizedependent increases in tumor $\mathrm{v}_{\mathrm{e}}$ in both tumor groups $(\mathrm{p}<$ 0.001 ), but in sporadic tumors this increase stopped once tumor volume exceeded $10 \mathrm{~cm}^{3}$ (Fig. 1B). Mean tumor $\mathrm{R} 1_{\mathrm{N}}$ decreased with increasing tumor size in both sporadic $($ rho $=-0.58, \mathrm{p}=0.003)$ and NF2-related (rho $=-0.86, \mathrm{p}$ $<0.001$ ) VSs (Fig. 1C). As shown in Fig. 1D, both sporadic and NF2-related VSs also demonstrated increases in tumor MD with increasing tumor size $(\mathrm{p}<0.001)$, and there was a significant inverse linear relationship between tumor MD and FA across both tumor cohorts ( $\leq \leq 0.002$; Supplementary Fig. S3). Representative images from a patient with a large sporadic tumor and a patient with a large NF2-related VS are shown in Fig. 1E and F, respectively.

Univariate regression analysis (Supplementary Table S1) demonstrated that the cube root transform of tumor size and annual adjusted tumor growth rate were significant independent predictors of mean tumor $\mathrm{K}^{\text {trans }}$ $(\mathrm{p}<0.001)$, mean tumor $\mathrm{v}_{\mathrm{e}}(\mathrm{p} \leq 0.02)$, mean tumor $\mathrm{R} 1_{\mathrm{N}}$ $(\mathrm{p}<0.001)$, mean tumor diffusivity $(\mathrm{p} \leq 0.001)$, and mean 
TABLE 2. Comparative table of patient demographics, tumor laterality, and tumor size for the patients included in the pathology study

\begin{tabular}{|c|c|c|c|c|}
\hline Variable & Sporadic VS $(n=17)$ & NF2-Related VS ( $n=12)$ & Mean Difference (95\% Cl) & p Value* \\
\hline Median age at operation (IQR), yrs & $49.4(41.3-55.8)$ & $32.8(27.1-42.8)$ & $-12.2(-21.3$ to -3.1$)$ & 0.01 \\
\hline \multicolumn{5}{|l|}{ Sex, $n(\%)$} \\
\hline Male & $7(41.2)$ & $6(50)$ & & 0.64 \\
\hline Female & $10(59)$ & $6(50)$ & & \\
\hline \multicolumn{5}{|l|}{ Tumor laterality, $n(\%)$} \\
\hline Lt & $10(59)$ & $5(42)$ & & 0.36 \\
\hline Rt & $7(41.2)$ & $7(58.3)$ & & \\
\hline \multicolumn{5}{|l|}{ Koos grade, $n(\%)$} \\
\hline II & $3(18)$ & $3(25)$ & & 0.39 \\
\hline III & $7(41)$ & $2(17)$ & & \\
\hline IV & $7(41)$ & $7(58)$ & & \\
\hline Median tumor volume (IQR), $\mathrm{cm}^{3}$ & $2.51(1.56-5.91)$ & $8.43(0.67-28.28)$ & $9.23(0.53$ to 17.9$)$ & 0.33 \\
\hline Median annual adjusted growth rate (IQR), $\mathrm{cm}^{3} / \mathrm{yr}$ & $0.77(0.54-2.71)$ & $0.80(0.46-1.78)$ & $0.23(-1.17$ to 1.65$)$ & 0.76 \\
\hline Median length of radiological follow-up (IQR), mos & $13(8-24)$ & $28(6-67)$ & $18(-2$ to 37$)$ & 0.35 \\
\hline
\end{tabular}

Boldface type indicates statistical significance.

* The p value was calculated using the Mann-Whitney U-test, or Pearson's chi-square test in the case of tumor laterality, sex, and tumor Koos grade.

tumor FA ( $\mathrm{p} \leq 0.003)$. Univariate and multiple regression analysis (Supplementary Table S2) demonstrated that with the exception of MD ( $\mathrm{p}<0.001)$, tumor NF2 status had no statistically significant effect on any of the imaging parameters or the observed relationship between the derived imaging parameters and tumor size $(p>0.05)$. Intertumor correlation analyses between different imaging metrics across each VS cohort are shown in Supplementary Tables S3 and S4.

\section{Imaging and Pathology Correlation Analysis}

Correlation indices between imaging and tissue analysis in the 17 sporadic VSs are shown in Supplementary Table S5. Cell density correlated negatively with mean
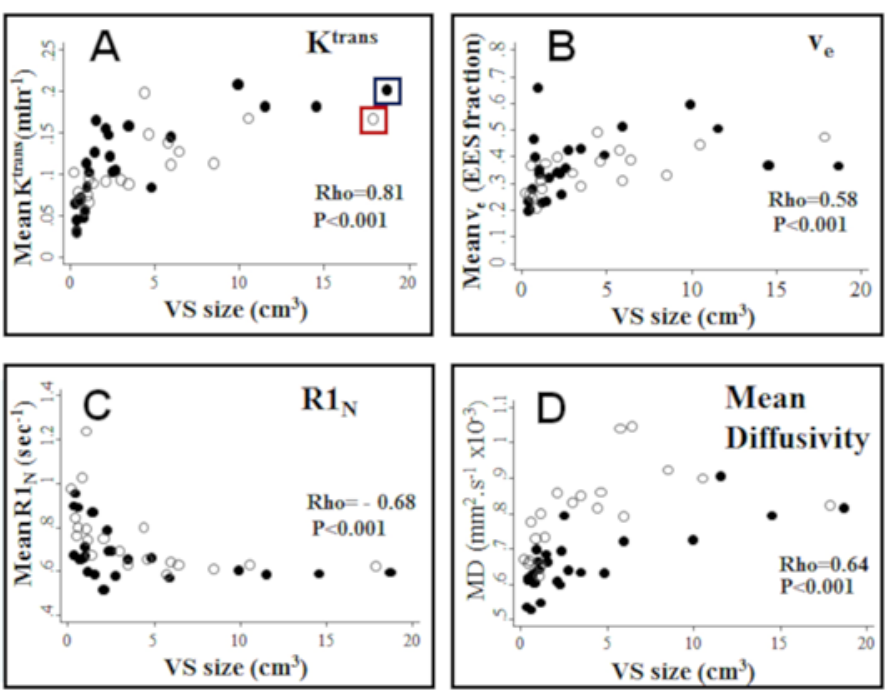
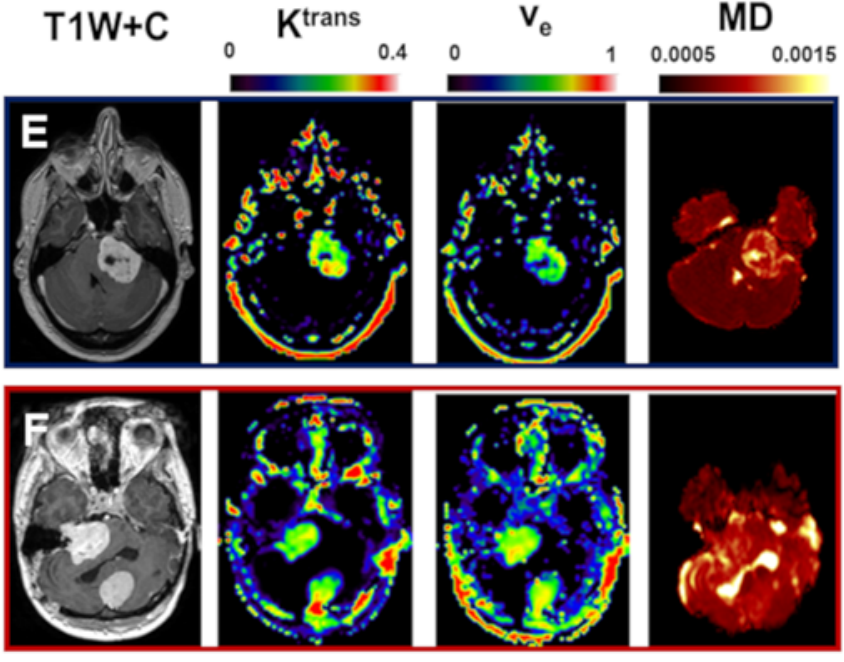

- Sporadic oNF2

FIG. 1. Comparison of DCE-MRI-derived microvascular parameters and diffusion metrics between sporadic and NF2-related VSs. A: Intertumor scatterplot analysis of mean tumor $K^{\text {trans }}$ against VS size $\left(\mathrm{cm}^{3}\right)$. B: Intertumor scatterplot analysis of mean tumor $\mathrm{V}_{\mathrm{e}}$ against VS size $\left(\mathrm{cm}^{3}\right)$. C: Intertumor scatterplot analysis of mean tumor R1 $\mathrm{N}$ against VS size $\left(\mathrm{cm}^{3}\right)$. D: Intertumor scatterplot analysis of tumor MD $\left(\mathrm{mm}^{2} \cdot \mathrm{s}^{-1} \times 10^{-3}\right)$ against VS size $\left(\mathrm{cm}^{3}\right)$. E and F: Parametric maps from a patient with a large sporadic tumor and a patient with a large NF2-related VS are shown in E and F, respectively. Mean parameter values for the VSs displayed in E and $F$ are outlined in the scatterplot shown in panel A through the use of a blue and red box, respectively. Spearman's rho reported for the intertumor correlation across all 44 VSs (A-D). EES = extravascular-extracellular space; T1W+C = T1-weighted postcontrast. Figure is available in color online only. 

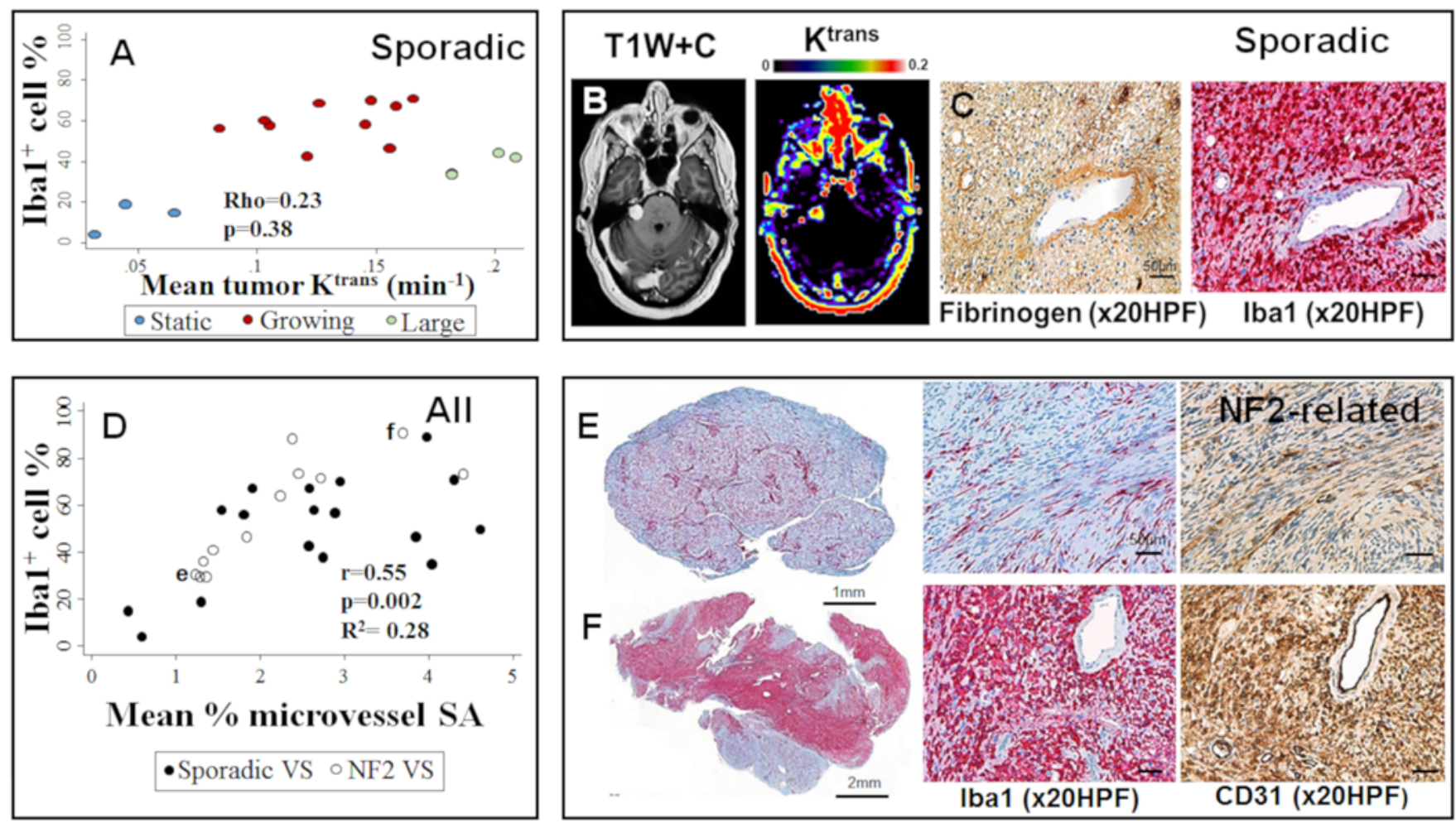

FIG. 2. Relationship between tumor vascularity and intratumoral inflammation in sporadic and NF2-related VSs. A: Intertumor scatterplot analysis of mean tumor $\mathrm{K}^{\text {trans }}$ against mean Iba1+ cell percentage in 17 resected sporadic VSs. Differences in Iba1 ${ }^{+}$ TAM density between the growing VSs (red), static VSs (blue), and large VSs that were primarily resected due to size (green) are shown. B: Representative image sections (left: T1-weighted postcontrast; right: parametric K ${ }^{\text {trans }}$ map) from a 55 -year-old female patient with a rapidly growing sporadic VS. C: Immunostains from the same tumor demonstrating high Iba1+ TAM density and corresponding regions of perivascular leak as demonstrated by fibrinogen immunostaining (immunoperoxidase-whole section). D: Intertumor scatterplot comparison of mean CD31 percentage microvessel SA against mean lba1+ cell percentage in 17 sporadic and 12 NF2-related VSs. E: Immunostains (Iba1 = red, CD31 = brown) from a slow-growing NF2-related VS (see labeled data point in panel D) demonstrating low Iba1+ TAM density and vascularity. F: Immunostains from a rapidly growing NF2-related VS (see labeled data point in panel D) demonstrating high Iba1+ TAM density and corresponding regions of high vascularity. Pearson's product-moment correlation coefficient ( $r$ ) reported, or Spearman's rho in the case of nonlinear association between variables. HPF = high-power field. Figure is available in color online only.

tumor $\mathrm{v}_{\mathrm{e}}($ rho $=-0.61, \mathrm{p}=0.02)$. Tumor MD correlated positively with fibrinogen OD (rho $=0.70, p=0.002$ ), but there was no correlation observed between cell density and either diffusion metric. Tumor $\mathrm{K}^{\text {trans }}$ correlated positively with both mean fibrinogen OD $(r=0.62, p=0.008)$ and microvessel SA $(r=0.74, p<0.001)$. Derived $v_{p}$ estimates correlated strongly with microvessel SA $(r=0.89$, $p$ $<0.001$; Supplementary Fig. S4).

The 10 rapidly growing VSs demonstrated higher TAM density compared to both the static $(\mathrm{p}<0.001)$ and large $(\mathrm{p}$ $=0.003$ ) VS groups (Supplementary Table S6). There was a nonsignificant increase in TAM infiltrates with increasing tumor $\mathrm{K}^{\text {trans }}$ across all tumors (rho $=0.23, \mathrm{p}=0.38$; Fig. 2A). The 4 large VSs demonstrated marked heterogeneity in TAM density, and after exclusion of these tumors the relationship between $\mathrm{Iba}^{+}$cell percentage and $\mathrm{K}^{\text {trans }}$ was statistically significant (rho $=0.76, p=0.002$ ). Compared to static tumors, growing VSs displayed significantly greater overall microvessel SA ( $p=0.011)$. Image sections from a growing VS with high $\mathrm{K}^{\text {trans }}$ values are shown in Fig. $2 \mathrm{~B}$ alongside comparative immunostains demonstrating the high vascular permeability and dense TAM infiltrates seen within this tumor (Fig. 2C). Across all VS tissue specimens there was a positive correlation between microvessel $\mathrm{SA}$ and $\mathrm{Iba}^{+}$TAM density $(\mathrm{r}=0.55, \mathrm{p}=0.002$; Fig. 2D). Representative tissue sections from both a slowly growing and rapidly growing NF2-related VS are shown in Fig. 2E and $\mathrm{F}$, respectively, and demonstrate the close correspondence between tumor vascularity and TAM infiltrates.

\section{Sporadic and NF2-Related VS Pathology Comparison}

As shown in Table 3, NF2-related VSs demonstrated significantly lower fibrinogen OD values $(\mathrm{p}<0.001)$ compared to growing sporadic VS; there was no significant difference in either TAM density $(p=0.65)$ or microvessel SA $(p=0.16)$. Univariate linear regression analysis to evaluate the effect of tumor size, tumor growth rate, and tumor NF2 status on tissue-derived parameters is shown in Supplementary Table S7. Whereas both tumor size and tumor growth were significant independent predictors of mean Iba1 ${ }^{+}$cell percentage $(\mathrm{p} \leq 0.04)$ and mean CD31 percentage microvessel SA ( $\leq \leq 0.01)$, with the exception of mean tissue fibrinogen OD $(\mathrm{p}=0.001)$, tumor NF2 status 
TABLE 3. Comparative tissue-derived parameters between sporadic and NF2-related VSs

\begin{tabular}{|c|c|c|c|c|c|c|c|}
\hline & A & $\mathrm{B}$ & $\mathrm{C}$ & C vs $A$ & & C vs B & \\
\hline Variable & $\begin{array}{c}\text { Sporadic VS, } \\
\text { All }(n=17)\end{array}$ & $\begin{array}{l}\text { Sporadic VS, } \\
\text { Growing }(n=10)\end{array}$ & $\begin{array}{l}\text { NF2-Related } \\
\text { VS }(n=12)\end{array}$ & $\begin{array}{l}\text { Mean Difference } \\
\quad(95 \% \mathrm{Cl})\end{array}$ & $\begin{array}{c}\mathrm{p} \\
\text { Value }^{*}\end{array}$ & $\begin{array}{l}\text { Mean Difference } \\
\quad(95 \% \mathrm{Cl})\end{array}$ & $\begin{array}{c}p \\
\text { Value* }\end{array}$ \\
\hline Mean Iba1+ cell \% & $46.5 \pm 20.2$ & $59.9 \pm 9.75$ & $56.3 \pm 23.2$ & $9.73(-6.88$ to 26.34$)$ & 0.24 & $-3.62(-20.05$ to 12.81$)$ & 0.65 \\
\hline Mean CD31 \% microvessel SA & $2.63 \pm 1.26$ & $2.81 \pm 0.96$ & $2.20 \pm 1.02$ & $-0.43(-1.33$ to 0.47$)$ & 0.33 & $-0.62(-1.50$ to 0.27$)$ & 0.16 \\
\hline $\begin{array}{l}\text { Cell density (H \& E cell nuclei/ } \\
20 \text { HPF) }\end{array}$ & $2823 \pm 906$ & $2401 \pm 865$ & $2272 \pm 621$ & $-550(-1170$ to 70$)$ & 0.08 & $-129(-790$ to 533$)$ & 0.68 \\
\hline Mean fibrinogen OD (\%) & $0.37 \pm 0.13$ & $0.37 \pm 0.08$ & $0.24 \pm 0.03$ & $-0.12(-0.20$ to -0.04$)$ & 0.004 & $-0.12(-0.18$ to -0.07$)$ & $<0.001$ \\
\hline
\end{tabular}

$\mathrm{HPF}=$ high-power field.

Data for $A, B$, and $C$ are given as mean $\pm S D$. Boldface type indicates statistical significance.

* The $p$ value was calculated using 1-way ANOVA with Bonferroni correction.

had no statistically significant effect on any of the tissue parameters.

\section{Relationship Between Microvessel SA, TAM Density, and Cellular Proliferation Indices}

Figure 3 demonstrates the relationship between TAM density, microvessel SA, and Ki-67 labeling index in the 4 large sporadic VSs. Both microvessel SA $(\mathrm{p}<0.001$;
Fig. 3A) and fibrinogen OD ( $p<0.001)$ were significantly elevated in areas of high TAM density compared to lowTAM-density regions (Supplementary Table S8). Across all tumor areas, TAM density correlated with fibrinogen $\mathrm{OD}(\mathrm{rho}=0.71, \mathrm{p}<0.001 ;$ Fig. 3B) and microvessel SA (rho $=0.52, \mathrm{p}<0.001)$. The Ki-67 labeling index was significantly greater in regions of high TAM density compared to low-density regions ( $<<0.001$; Fig. 3C). A whole-mount
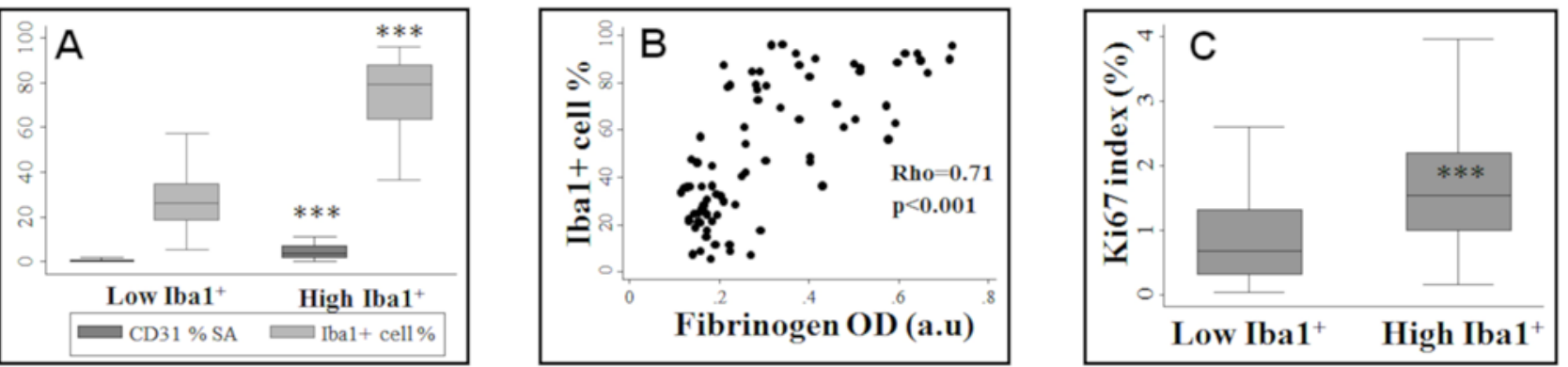

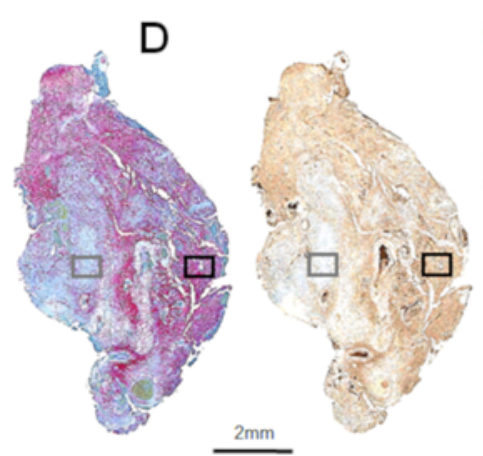

Iba1

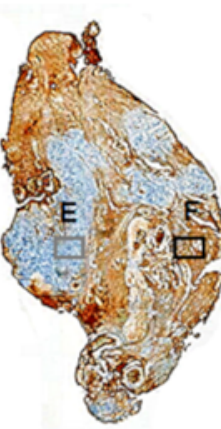

Fibrinogen

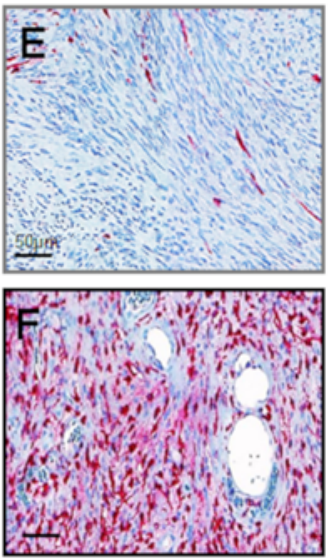

Iba1 (x20HPF)

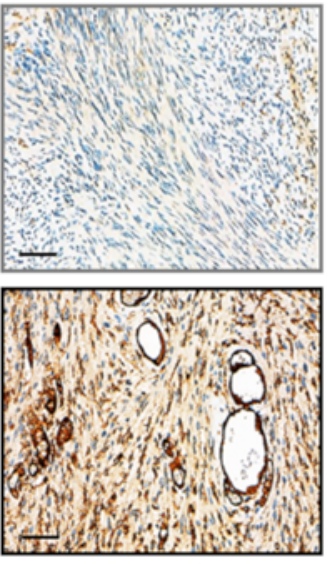

CD31 (x20HPF)

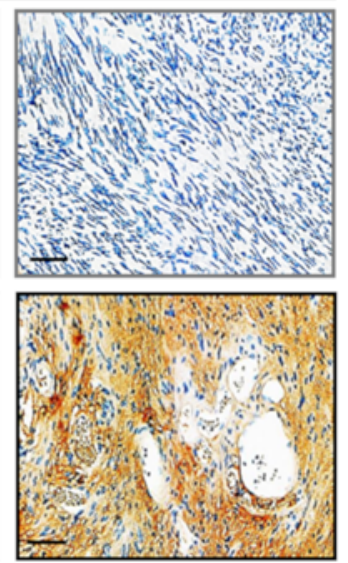

Fibrinogen (x20HPF)

FIG. 3. Intratumoral relationship between microvessel SA, TAM density, and cellular proliferation indices. A: Boxplot comparison of CD31 percentage microvessel SA and Iba1 ${ }^{+}$cell percentage per $\times 20$ HPF within areas of low and high TAM density. Data shown for 4 sporadic VSs (2-tailed t-test, $\left.{ }^{* *} p<0.001\right)$. B: Scatterplot comparison of fibrinogen OD and Iba1 ${ }^{+}$cell percentage across 80 different tumor regions (20 regions per tumor). Spearman's rho reported. C: Boxplot comparison of mean Ki-67 labeling index across high and low Iba1 ${ }^{+}$TAM areas (2-tailed t-test, $\left.{ }^{* *} \mathrm{p} \leq 0.001\right)$. D: Immunostains (Iba1 $=$ red, CD31 = brown, fibrinogen = brown) from a large sporadic VS demonstrating colocalization between TAM infiltrates, regions of high vascularity, and perivascular leak, as demonstrated by fibrinogen immunostaining (immunoperoxidase-whole section). E and F: Higher-magnification images ( $\times 20 \mathrm{HPF}$ ) of the areas framed in the whole mounts (immunoperoxidase, $\times 20$ ) demonstrating a region of low (E) and high (F) TAM density, respectively. Figure is available in color online only. 
A

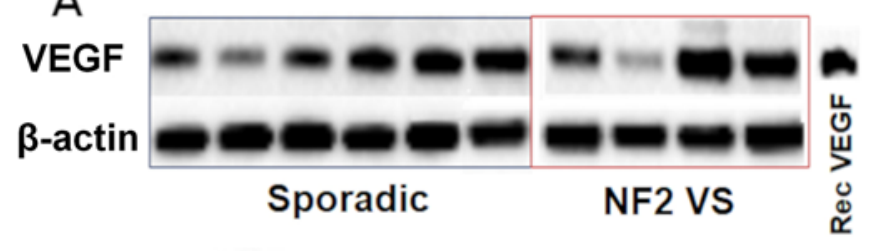

B

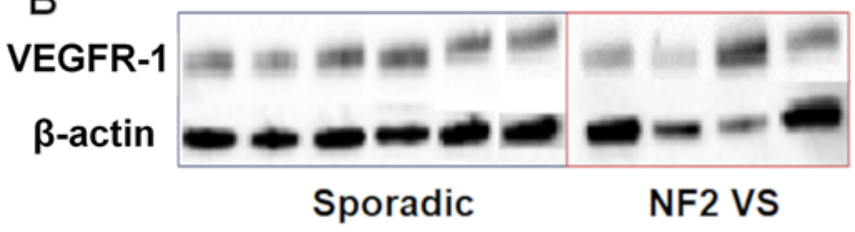

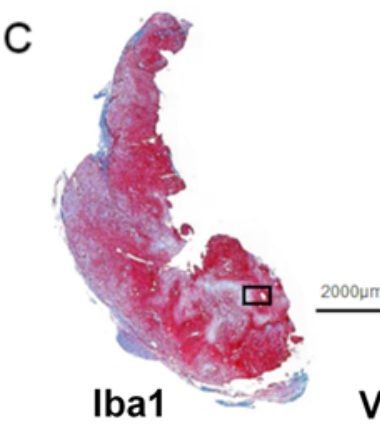

Iba1

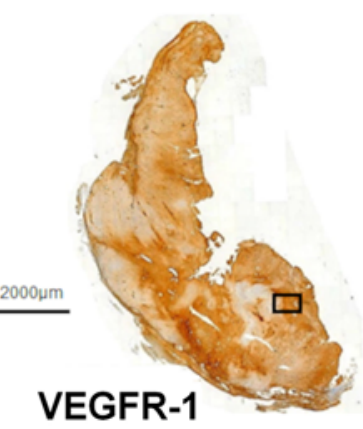

VEGFR-1

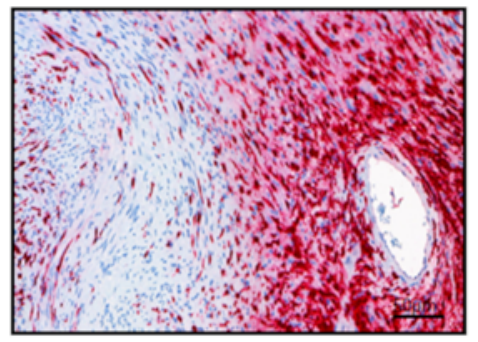

Iba1 (x20HPF)

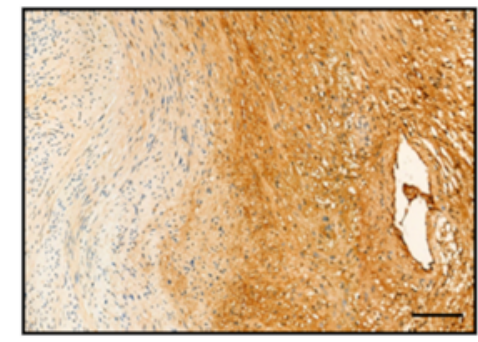

VEGFR-1 (x20HPF)

FIG. 4. Expression of VEGF and VEGFR-1 in VS. A: Western blot demonstrating VEGF protein expression in a cohort of sporadic and NF2-related VSs, with $\beta$ actin as a control. B: Western blot demonstrating VEGFR-1 protein expression in a cohort of sporadic and NF2-related VSs with $\beta$ actin as a control. C: Immunostains (VEGFR-1 = brown, Iba1 = red) from a growing sporadic VS demonstrating spatial colocalization of VEGFR-1 and lba1 immunostaining (left = immunoperoxidase- whole section, right $=\mathrm{im}$ munoperoxidase, $x 20 \mathrm{HPF})$. Figure is available in color online only.

section from a large sporadic VS with corresponding regions of both high and low TAM density, microvessel SA, and vascular leak is shown in Fig. 3D-F.

\section{Tumor VEGF and VEGFR-1 Expression}

VEGF expression was present in all sporadic and NF2related VSs (Fig. 4A). Western blot results confirmed the immunohistochemistry data. The molecular weight of VEGF was approximately $20 \mathrm{KDa}$. VEGF expression varied across individual cases, but there were no statistically significant differences between sporadic and NF2related VSs ( $p=0.21$, Fisher's exact test). VEGFR-1 was expressed in all cases. Western blot analysis confirmed the expression (Fig. 4B) and showed a molecular weight of approximately $65 \mathrm{KDa}$, consistent with the soluble isoform of the VEGFR-1. ${ }^{27-29}$ Sporadic and NF2-related VSs showed spatial correspondence of areas of high TAM density and VEGFR-1 expression (Fig. 4C). Spatial correspondence was also found between areas of high TAM density and VEGF expression (Fig. 5A and B). Double immunofluorescence demonstrated that VEGF was expressed mostly by TAMs (Fig. 5C), although the intensity of VEGF staining varied from area to area. We were unable to confirm the expression of VEGFR-1 in TAMs due to the low signal of VEGFR-1 in immunofluorescence.

\section{Discussion}

This study expanded on our previous observation showing that an increase in inflammation and vascular permeability correlates with growth in sporadic VSs. ${ }^{8}$ The present findings demonstrate that sporadic and NF2-related VSs show similar microvasculature and a similar relationship between neovascularization and intratumoral inflammation, and that VEGF-expressing TAMs are instrumental to angiogenesis. Our results also suggest that inflamma- tion and angiogenesis can represent potential therapeutic targets in both tumor groups.

To our knowledge, this is the first study that compared DCE-MRI and diffusion MRI-derived microvascular and microstructural metrics in sporadic and NF2-related VSs. Alongside increases in tumor $\mathrm{K}^{\text {trans }}$, both sporadic and NF2-related VSs demonstrated concomitant increases in the extravascular-extracellular space fraction $\left(\mathrm{v}_{\mathrm{e}}\right)$ and reductions in $\mathrm{R} 1_{\mathrm{N}}$, an inverse correlate of tumoral free water content ${ }^{23}$ with increasing tumor size. Derived diffusion metrics mirrored these changes and supported previous evidence $^{30}$ that larger tumors display both higher MD and lower FA, a DTI-derived diffusion directionality measure sensitive to changes in tumor organization and microstructure. $^{31,32}$ Our imaging findings suggest that as both sporadic and NF2-related VSs grow and enlarge, there are not only increases in the capillary permeability SA product, but also concomitant increases in the extravascular-extracellular space and the degree of interstitial tumoral free water, and a reduction in the overall density of the tissue microstructural architecture.

Our results demonstrated a relationship between tumor vascularity and TAM infiltration and showed that DCE-MRI-derived vascular biomarkers such as $\mathrm{K}^{\text {trans }}$ can be used as indirect measures of inflammatory response, although a direct comparison between imaging data and tissue analysis was not feasible in the NF2 cohort. Nonetheless, analysis of a separate cohort of resected growing NF2-related VSs showed a strong correlation between TAMs and microvessel SA, similar to what was observed in sporadic VS.

The 4 large sporadic VSs in our cohort demonstrated disproportionately high $\mathrm{K}^{\text {trans }}$ values compared to the average TAM tissue density. The discrepancy between imaging and tissue data may result from limitations of our DCE-MRI technique when applied to highly vascular 
A
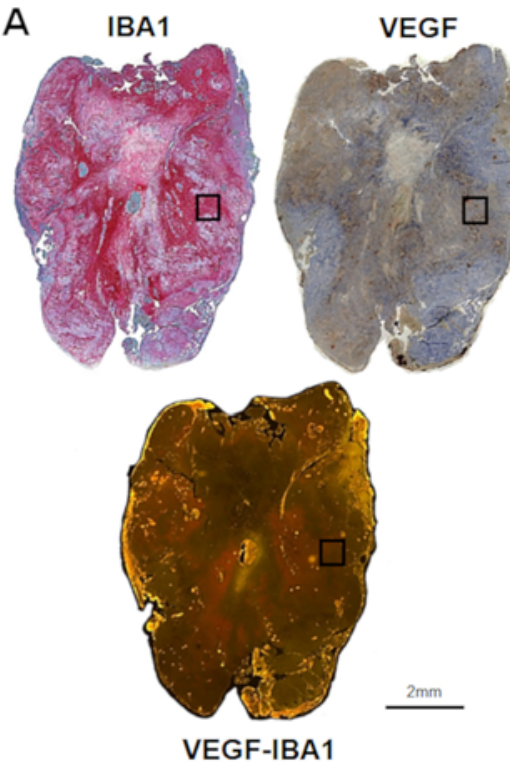

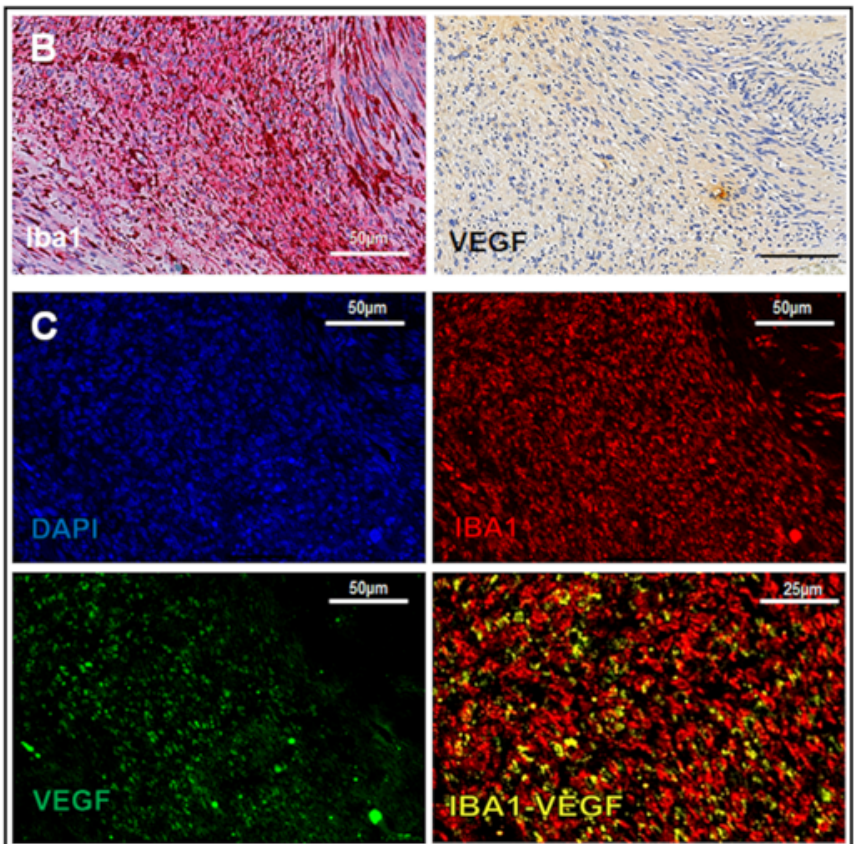

FIG. 5. Immunostains demonstrating Iba1 and VEGF coexpression. A: Immunostains from a rapidly growing sporadic VS demonstrating macroscopic spatial colocalization between Iba1 and VEGF: upper left = Iba1 (red), immunoperoxidase-whole section; upper right = VEGF (brown), immunoperoxidase - whole section; lower = immunofluorescence demonstrating colocalization between Iba1 (red channel) and VEGF (green channel). B: Higher magnification ( $\times 20 \mathrm{HPF})$ of the area framed in the whole sections $(\mathrm{A}$; immunoperoxidase, $\times 20$ ) demonstrates a region of high lba1+ TAM density (left) with corresponding immunopositivity for VEGF (right). C: Higher magnification ( $\times 20 \mathrm{HPF}$ ) immunofluorescence images of the area framed in the whole sections demonstrates areas of cellular colocalization between Iba1 and VEGF (yellow): DAPI (blue), Iba1 (red), VEGF (green), and Iba1-VEGF (red-green; immunofluorescence, $\times 20$ ). Figure is available in color online only.

tumors, but may also reflect the dramatic differences in spatial resolution between imaging and tissue-based techniques. Across these tumors, there was considerable heterogeneity in TAM density, with regions of both high and low vascularity and TAM infiltration, respectively, and the spatial resolution of our DCE-MRI-derived metrics may limit the ability to fully detect such heterogeneity in vivo. In a previous study it was demonstrated that within growing sporadic VSs, macrophages rather than Schwann cells accounted for the majority of proliferating cells. ${ }^{8}$ Our demonstration of elevated cellular proliferation rates within intratumoral regions of high TAM density further supports this finding, and suggests that even within individual tumors, growth occurs within regions of high vascularity and high TAM infiltration.

Previous studies have documented the proangiogenic cytokine VEGF and VEGFR-1 in VS tissue,,$^{17,18,33}$ but the cellular origin of these proteins and their relationship to intratumoral inflammation were not investigated. We demonstrated that intratumoral macrophages contribute significantly to VEGF production, and we showed predominant expression of the soluble form of the VEGFR-1. ${ }^{27,29,34}$ Alongside its roles in promoting vasodilatation, increasing vascular permeability, and inducing angiogenesis, ${ }^{17,35}$ VEGF isoforms act as a chemoattractant for circulating VEGFR-1-expressing macrophages. ${ }^{35-38}$ Combined imaging and pathology results presented here further support our previous results of increased inflammation and vascular permeability in growing sporadic VSs, ${ }^{10}$ but also suggest that VEGF/VEGFR-1 signaling drives chemoattraction of VEGFR-1-expressing macrophages into the TME, ${ }^{36,37}$ in addition to driving angiogenesis. Further work is required to better evaluate the role that VEGFR-1-expressing macrophages play in VS angiogenesis and progression.

Among NF2 patients, the management of VS presents the biggest treatment challenge. Their bilateral and progressive nature causes considerable patient morbidity and makes treatment of these lesions through surgery or radiotherapy difficult. ${ }^{6}, 16$ To date, the anti-VEGF antibody bevacizumab is the only targeted molecular therapy for these tumors, ${ }^{19,20}$ but concerns have been raised about its widespread usage due to cardiovascular and renal complications and the duration of response. ${ }^{39,40}$ Our evidence of a spatial relationship between TAM and angiogenesis in sporadic and NF2-related VSs suggests that treatment targeting VEGF may lead to a concomitant reduction in TAM and therefore further impact tumor growth. The fact that TAM rather than tumor cells proliferates in $\mathrm{VS}^{8}$ further supports the idea of targeting macrophages along with vascular supply. Recent studies have documented upregulation of other proinflammatory cytokines/chemokines such as interelukin-1 $\beta$, interleukin-6, tumor necrosis factor- $\alpha$, and macrophage colony-stimulating factor in sporadic VS. ${ }^{41-43}$ A number of safe, effective, and well-tolerated immunomodulatory agents are already in clinical usage against these molecules, and our findings raise the possibility that these agents could be repurposed to help control tumor progression and growth in both VS groups. 


\section{Conclusions}

This is the largest comparative study of MRI-derived microstructural and microvascular characteristics in both sporadic and NF2-related VSs and the first such study to compare, in tissue, the relationship between tumor vascularity and inflammatory cell infiltration in both these tumor groups. Our imaging and tissue findings have demonstrated that both sporadic and NF2-related VSs show marked similarities regarding their microvascular metrics, and that in both tumor groups inflammation may be a relevant therapeutic target.

\section{Acknowledgments}

We thank Roger Meadows from the University of Manchester Bioimaging Facility for his help with the microscopy. We would also like to thank Dr. Damien McHugh for his advice on the diffusion MR analysis and Dr. Calvin Heal of the University of Manchester, Centre for Biostatistics, for his advice on the statistical approach. The work was supported by funding from the CRUK Cancer Imaging Centre in Cambridge and Manchester (grant no. C8742/A18097) and the Dowager Countess Eleanor Peel Trust. S.K.L. and D.G.E. are supported by the NIHR Biomedical Research Centre Manchester (grant no. IS-BRC-1215-20007).

\section{References}

1. Evans DG, Howard E, Giblin C, et al. Birth incidence and prevalence of tumor-prone syndromes: estimates from a UK family genetic register service. Am J Med Genet A. 2010;152A(2):327-332.

2. Hadfield KD, Smith MJ, Urquhart JE, et al. Rates of loss of heterozygosity and mitotic recombination in NF2 schwannomas, sporadic vestibular schwannomas and schwannomatosis schwannomas. Oncogene. 2010;29(47):6216-6221.

3. Evans DGR. Neurofibromatosis type 2 (NF2): a clinical and molecular review. Orphanet J Rare Dis. 2009;4(1):16.

4. Lallemand D, Manent J, Couvelard A, et al. Merlin regulates transmembrane receptor accumulation and signaling at the plasma membrane in primary mouse Schwann cells and in human schwannomas. Oncogene. 2009;28(6):854-865.

5. Evans DGR, Stivaros SM. Multifocality in neurofibromatosis type 2. Neuro Oncol. 2015;17(4):481-482.

6. Stivaros SM, Stemmer-Rachamimov AO, Alston R, et al. Multiple synchronous sites of origin of vestibular schwannomas in neurofibromatosis Type 2. J Med Genet. 2015;52(8):557-562.

7. Carlson ML, Link MJ, Wanna GB, Driscoll CLW. Management of sporadic vestibular schwannoma. Otolaryngol Clin North Am. 2015;48(3):407-422.

8. Lewis D, Roncaroli F, Agushi E, et al. Inflammation and vascular permeability correlate with growth in sporadic vestibular schwannoma. Neuro Oncol. 2019;21(3):314-325.

9. de Vries M, Briaire-de Bruijn I, Malessy MJA, et al. Tumorassociated macrophages are related to volumetric growth of vestibular schwannomas. Otol Neurotol. 2013;34(2):347-352.

10. de Vries M, Hogendoorn PCW, Briaire-de Bruyn I, et al. Intratumoral hemorrhage, vessel density, and the inflammatory reaction contribute to volume increase of sporadic vestibular schwannomas. Virchows Arch. 2012;460(6):629-636.

11. Schulz A, Büttner R, Hagel C, et al. The importance of nerve microenvironment for schwannoma development. Acta Neuropathol. 2016;132(2):289-307.

12. Wang S, Liechty B, Patel S, et al. Programmed death ligand 1 expression and tumor infiltrating lymphocytes in neurofibromatosis type 1 and 2 associated tumors. J Neurooncol. 2018;138(1):183-190.
13. West N, Sass H, Cayé-Thomasen P. Sporadic and NF2associated vestibular schwannoma surgery and simultaneous cochlear implantation: a comparative systematic review. Eur Arch Otorhinolaryngol. 2020;277(2):333-342.

14. Lassaletta L, Aristegui M, Medina M, et al. Ipsilateral cochlear implantation in patients with sporadic vestibular schwannoma in the only or best hearing ear and in patients with NF2. Eur Arch Otorhinolaryngol. 2016;273(1):27-35.

15. Moffat DA, Lloyd SKW, Macfarlane R, et al. Outcome of translabyrinthine surgery for vestibular schwannoma in neurofibromatosis type 2. Br J Neurosurg. 2013;27(4):446-453.

16. Evans DG, Baser ME, O'Reilly B, et al. Management of the patient and family with neurofibromatosis 2: a consensus conference statement. Br J Neurosurg. 2005;19(1):5-12.

17. Cayé-Thomasen P, Werther K, Nalla A, et al. VEGF and VEGF receptor-1 concentration in vestibular schwannoma homogenates correlates to tumor growth rate. Otol Neurotol. 2005;26(1):98-101.

18. Koutsimpelas D, Stripf T, Heinrich UR, et al. Expression of vascular endothelial growth factor and basic fibroblast growth factor in sporadic vestibular schwannomas correlates to growth characteristics. Otol Neurotol. 2007;28(8):1094-1099.

19. Plotkin SR, Merker VL, Halpin C, et al. Bevacizumab for progressive vestibular schwannoma in neurofibromatosis type 2: a retrospective review of 31 patients. Otol Neurotol. 2012;33(6):1046-1052.

20. Plotkin SR, Stemmer-Rachamimov AO, Barker FG II, et al Hearing improvement after bevacizumab in patients with neurofibromatosis type 2. N Engl J Med. 2009;361(4):358367.

21. Tofts PS, Brix G, Buckley DL, et al. Estimating kinetic parameters from dynamic contrast-enhanced $\mathrm{T}_{1}$-weighted MRI of a diffusable tracer: standardized quantities and symbols. $J$ Magn Reson Imaging. 1999;10(3):223-232.

22. Haris M, Husain N, Singh A, et al. Dynamic contrast-enhanced derived cerebral blood volume correlates better with leak correction than with no correction for vascular endothelial growth factor, microvascular density, and grading of astrocytoma. J Comput Assist Tomogr. 2008;32(6):955-965.

23. Li KL, Djoukhadar I, Zhu X, et al. Vascular biomarkers derived from dynamic contrast-enhanced MRI predict response of vestibular schwannoma to antiangiogenic therapy in type 2 neurofibromatosis. Neuro Oncol. 2016;18(2):275-282.

24. Li K-L, Buonaccorsi G, Thompson G, et al. An improved coverage and spatial resolution-using dual injection dynamic contrast-enhanced (ICE-DICE) MRI: a novel dynamic contrast-enhanced technique for cerebral tumors. Magn Reson Med. 2012;68(2):452-462.

25. Smith SM. Fast robust automated brain extraction. Hum Brain Mapp. 2002;17(3):143-155.

26. Li KL, Lewis D, Jackson A, et al. Low-dose T1W DCE-MRI for early time points perfusion measurement in patients with intracranial tumors: a pilot study applying the microsphere model to measure absolute cerebral blood flow. J Magn Reson Imaging. 2018;48(2):543-557.

27. Sela S, Natanson-Yaron S, Zcharia E, et al. Local retention versus systemic release of soluble VEGF receptor-1 are mediated by heparin-binding and regulated by heparanase. Circ Res. 2011;108(9):1063-1070.

28. Kendall RL, Wang G, Thomas KA. Identification of a natural soluble form of the vascular endothelial growth factor receptor, FLT-1, and its heterodimerization with KDR. Biochem Biophys Res Commun. 1996;226(2):324-328.

29. Roeckl W, Hecht D, Sztajer H, et al. Differential binding characteristics and cellular inhibition by soluble VEGF receptors 1 and 2. Exp Cell Res. 1998;241(1):161-170.

30. Sener RN. Diffusion magnetic resonance imaging of solid vestibular schwannomas. J Comput Assist Tomogr. 2003;27(2):249-252. 
31. Bammer R. Basic principles of diffusion-weighted imaging. Eur J Radiol. 2003;45(3):169-184.

32. Alexander AL, Lee JE, Lazar M, Field AS. Diffusion tensor imaging of the brain. Neurotherapeutics. 2007;4(3):316-329.

33. Cayé-Thomasen P, Baandrup L, Jacobsen GK, et al. Immunohistochemical demonstration of vascular endothelial growth factor in vestibular schwannomas correlates to tumor growth rate. Laryngoscope. 2003;113(12):2129-2134.

34. Ambati BK, Nozaki M, Singh N, et al. Corneal avascularity is due to soluble VEGF receptor-1. Nature. 2006;443(7114):993-997.

35. Ferrara N, Gerber HP, LeCouter J. The biology of VEGF and its receptors. Nat Med. 2003;9(6):669-676.

36. Weddell JC, Chen S, Imoukhuede PI. VEGFR1 promotes cell migration and proliferation through PLC $\gamma$ and PI3K pathways. NPJ Syst Biol Appl. 2017;4(1):1.

37. Freire Valls A, Knipper K, Giannakouri E, et al. VEGFR1 ${ }^{+}$ metastasis-associated macrophages contribute to metastatic angiogenesis and influence colorectal cancer patient outcome. Clin Cancer Res. 2019;25(18):5674-5685.

38. Kerber M, Reiss Y, Wickersheim A, et al. Flt-1 signaling in macrophages promotes glioma growth in vivo. Cancer Res. 2008;68(18):7342-7351.

39. Blakeley JO, Evans DG, Adler J, et al. Consensus recommendations for current treatments and accelerating clinical trials for patients with neurofibromatosis type 2 . Am J Med Genet A. 2012;158A(1):24-41.

40. Mautner V-F, Nguyen R, Knecht R, Bokemeyer C. Radiographic regression of vestibular schwannomas induced by bevacizumab treatment: sustain under continuous drug application and rebound after drug discontinuation. Ann Oncol. 2010;21(11):2294-2295.

41. Taurone S, Bianchi E, Attanasio G, et al. Immunohistochemical profile of cytokines and growth factors expressed in vestibular schwannoma and in normal vestibular nerve tissue. Mol Med Rep. 2015;12(1):737-745.

42. Breun M, Schwerdtfeger A, Martellotta DD, et al. CXCR4: a new player in vestibular schwannoma pathogenesis. Oncotarget. 2018;9(11):9940-9950.

43. de Vries WM, Briaire-de Bruijn IH, van Benthem PPG, et al. M-CSF and IL-34 expression as indicators for growth in sporadic vestibular schwannoma. Virchows Arch. 2019;474(3):375-381.
44. Koos WT, Day JD, Matula C, Levy DI. Neurotopographic considerations in the microsurgical treatment of small acoustic neurinomas. J Neurosurg. 1998;88(3):506-512.

\section{Disclosures}

Dr. Evans reports being a consultant to AstraZeneca.

\section{Author Contributions}

Conception and design: Lewis, Jackson, King, Coope. Acquisition of data: Lewis, Donofrio, O'Leary, Williams, Djoukhadar, Agushi, Stapleton, Lloyd, Freeman, Rutherford, HammerbeckWard, Evans, Jackson, Pathmanaban. Analysis and interpretation of data: Lewis, Donofrio, O'Leary, Li, Zhu, Williams, Agushi, Hannan, Roncaroli, Coope. Drafting the article: Lewis, Donofrio, O’Leary, Hannan, Roncaroli, King, Coope. Critically revising the article: Lewis, Donofrio, O'Leary, Li, Zhu, Williams, Hannan, Lloyd, Freeman, Wadeson, Rutherford, Hammerbeck-Ward, Evans, Jackson, Pathmanaban, Roncaroli, King, Coope. Reviewed submitted version of manuscript: all authors. Approved the final version of the manuscript on behalf of all authors: Lewis. Statistical analysis: Lewis, Donofrio, O'Leary. Administrative/technical/ material support: Wadeson. Study supervision: Jackson, King, Coope.

\section{Supplemental Information \\ Online-Only Content}

Supplemental material is available with the online version of the article.

Supplementary Methods, Tables, and Figures. https://thejns. org/doi/suppl/10.3171/2020.3.JNS193230.

\section{Correspondence}

Daniel Lewis: Manchester Centre for Clinical Neurosciences, Salford Royal NHS Foundation Trust, Manchester Academic Health Science Centre, Salford, Greater Manchester, UK. dan. lewis1112@gmail.com. 